\title{
Utility of feeding jejunostomy in patients with esophageal cancer undergoing esophagectomy with a high risk of anastomotic leakage
}

\author{
Weitao Zhuang ${ }^{1,2 \#} \wedge$, Hansheng $W^{1,3 \#}$, Huiling Liu ${ }^{1}$, Shujie Huang ${ }^{1,2}$, Yinghong $W^{1}{ }^{1}$, Cheng Deng ${ }^{1}$, \\ Dan Tian ${ }^{1}$, Zihao Zhou ${ }^{1}$, Ruiqing Shi ${ }^{1}$, Gang Chen ${ }^{1}$, Guillaume Piessen ${ }^{4}$, Puja G. Khaitan ${ }^{5}$, \\ Kazuo Koyanagi ${ }^{6}$, Soji Ozawa ${ }^{6}$, Guibin Qiao ${ }^{1 \wedge}$
}

${ }^{1}$ Department of Thoracic Surgery, Guangdong Provincial People's Hospital, Guangdong Academy of Medical Sciences, Guangzhou, China; ${ }^{2}$ Shantou University Medical College, Shantou, China; ${ }^{3}$ The Second School of Clinical Medicine, Southern Medical University, Guangzhou, China; ${ }^{4}$ University of Lille, Department of Digestive and Oncological Surgery, Claude Huriez University Hospital, Lille, France; ${ }^{5}$ Division of Thoracic and Esophageal Surgery, Department of Surgery, Georgetown University School of Medicine, MedStar Washington Hospital Center, Washington, DC, USA; ${ }^{6}$ Department of Gastroenterological Surgery, Tokai University School of Medicine, Isehara, Japan

Contributions: (I) Conception and design: W Zhuang, H Wu; (II) Administrative support: G Qiao; (III) Provision of study materials or patients: C Deng, D Tian, Z Zhou, R Shi, G Chen; (IV) Collection and assembly of data: H Wu, H Liu, S Huang; (V) Data analysis and interpretation: W Zhuang, S Huang; (VI) Manuscript writing: All authors; (VII) Final approval of manuscript: All authors.

\#These authors contributed equally to this work.

Correspondence to: Guibin Qiao, MD, PhD. Department of Thoracic Surgery, Guangdong Provincial People's Hospital, Guangdong Academy of Medical Sciences, 106 Zhongshan Second Road, Guangzhou 510080, China. Email: guibinqiao@126.com.

\begin{abstract}
Background: Feeding jejunostomy is widely used for enteral nutrition (EN) after esophagectomy; however, its risks and benefits are still controversial. We aimed to evaluate the short-term and long-term outcomes of feeding jejunal tube (FJT) in patients undergoing esophagectomy for esophageal squamous cell carcinoma (ESCC) who were deemed high-risk for anastomotic leakage.
\end{abstract}

Methods: We retrospectively analyzed 716 patients who underwent esophagectomy with (FJT group, n=68) or without (control group, $\mathrm{n}=648$ ) intraoperative placement of FJT. Propensity score matching (PSM) was used for the adjustment of confounding factors. Risk level for anastomotic leakage was determined for every patient after PSM.

Results: Patients in the FJT group were at higher risk of anastomotic leakage (14.9\% vs. $11.3 \%$ ), and had a statistically non-significant increase of postoperative complications $[31.3 \%$ vs. $21.8 \%$, odds ratio (OR) $=1.139,95 \%$ confidence interval (CI), 0.947-1.370, P=0.141] after PSM. Medical expenditure, length of postoperative hospital stay, and short-term mortality were similar between the FJT and control groups. Placement of FJT appeared to accelerate the recovery of anastomotic leakage (27.2 vs. $37.4 \mathrm{~d}, \mathrm{P}=0.073)$. Patients in FJT group achieved comparable overall survival (OS) both before [hazard ratio $(\mathrm{HR})=0.850$, $\mathrm{P}=0.390]$ and after $(\mathrm{HR}=0.797, \mathrm{P}=0.292)$ PSM.

Conclusions: FJT showed acceptable safety profile along with potential benefits for ESCC patients with a high presumed risk of anastomotic leakage. While FJT does not impact OS, placement of FJT should be considered in esophagectomy patients and tailored to individual patients based on their leak-risk profile.

Keywords: Esophageal cancer (ESCA); esophagectomy; feeding jejunostomy; anastomotic leakage

Submitted Sep 16, 2020. Accepted for publication Apr 09, 2021.

doi: 10.21037/jgo-21-133

View this article at: http://dx.doi.org/10.21037/jgo-21-133

^ ORCID: Weitao Zhuang, 0000-0002-8679-4109; Guibin Qiao, 0000-0001-9200-9317. 


\section{Introduction}

Esophagectomy is one of the most challenging procedures in oncology and one that is fraught with a high incidence of complications and a substantial decrease in health-related quality of life (HRQoL) $(1,2)$. At the current stage, nil by mouth in the first week after esophagectomy is generally applied in many institutions. However, postoperative weight loss is usually observed in these patients, which is an unfavorable prognostic factor (3). With the emerging concept of fast-track surgery, many institutions have attempted to implement the protocol of early oral feeding (EOF) after esophagectomy $(4,5)$. However, anastomotic leakage and aspiration pneumonia remain the two major concerns precluding postoperative $\operatorname{EOF}(5,6)$. In these circumstances, perioperative application of traditional methods, such as feeding jejunostomy, is still in demand to address the prevalent nutritional problems and allow the anastomosis to heal without additional strain from oral ingestion at early postoperative stage.

Intraoperative placement of feeding jejunal tube (FJT) offers a route for enteral nutrition (EN) to bridge the very first postoperative period to a safer stage for oral diet. Despite its extensive use and popularity, the risks and benefits of FJT in patients with esophageal cancer (ESCA) remain controversial (7-11). In prior studies, FJT has been associated with reduced length of hospital stay, and possibly with improved short-term mortality $(8,12)$. In the presence of anastomotic leakage, a lower risk for severe morbidity was also observed in patients with FJT (13). However, an increased risk of tube-related complications, such as superficial infection, bowel obstruction, intestinal torsion, and necrosis have been reported in many studies $(7,12,14,15)$, which might impair the HRQoL, and ultimately, the long-term survival outcome of the patients. Previous studies have mainly focused on the short-term outcomes of FJT $(7,8,11,14)$, while few studies have investigated its long-term prognostic impact (16). Additionally, no study to date has been conducted to investigate the effect of FJT in the specific group of patients with a higher pretest probability of anastomotic leakage. In other word, whether the addition of a FJT to the high risk group helped by decreasing the risk to a normal risk requires further investigation.

We carried out a retrospective study using a singleinstitution database of esophageal squamous cell carcinoma (ESCC). To the best of our knowledge, this is the first study using propensity score matching (PSM) to simultaneously evaluate the short-term effect and long-term survival outcome of FJT in the same group of patients. Preoperative risk assessment of anastomotic leakage was innovatively performed to analyze the effect of FJT on high risk patients. We aimed to address the abovementioned question and provide more evidence for clinical decision-making in placing FJT in patients with resectable ESCC. We present the following article in accordance with the STROBE reporting checklist (available at http://dx.doi.org/10.21037/ jgo-21-133).

\section{Methods}

\section{Patients}

A prospectively maintained database of ESCC in Guangdong Provincial People's Hospital was used to identify the study cohort. Clinical data in this database were continuously collected from the electronic medical records (EMR) according to a predefined protocol and had undergone regular quality control. All included patients had been treated for ESCC in our institution between January 2008 and December 2017. Patients who did not receive esophagectomy $(n=361)$, or had only pathologically confirmed epithelial dysplasia or high-grade intraepithelial neoplasia $(\mathrm{n}=5)$ were excluded for statistical analysis. A total of 716 out of 1,082 patients were finally eligible for the retrospective analyses (Figure 1). All included patients were restaged according to the $8^{\text {th }}$ edition of tumor-nodemetastasis (TNM) staging system. This study was approved by the Institutional Review Board of Guangdong Provincial People's Hospital (No. GDREC2019687H). All procedures performed in this study involving human participants were in accordance with the Declaration of Helsinki (as revised in 2013). Individual consent for this retrospective analysis was waived.

\section{Preoperative workup and neoadjuvant therapies}

All patients had routinely undergone comprehensive preoperative examinations, including esophageal endoscopy, barium swallow radiography, computed tomography (CT), cardiopulmonary function test, and biochemical blood tests. Neoadjuvant chemotherapy with a regimen of platinum plus paclitaxel was administered to select patients according to National Comprehensive Cancer Network (NCCN) guidelines. Neoadjuvant chemoradiation was not a preferential strategy in our institute, which was 


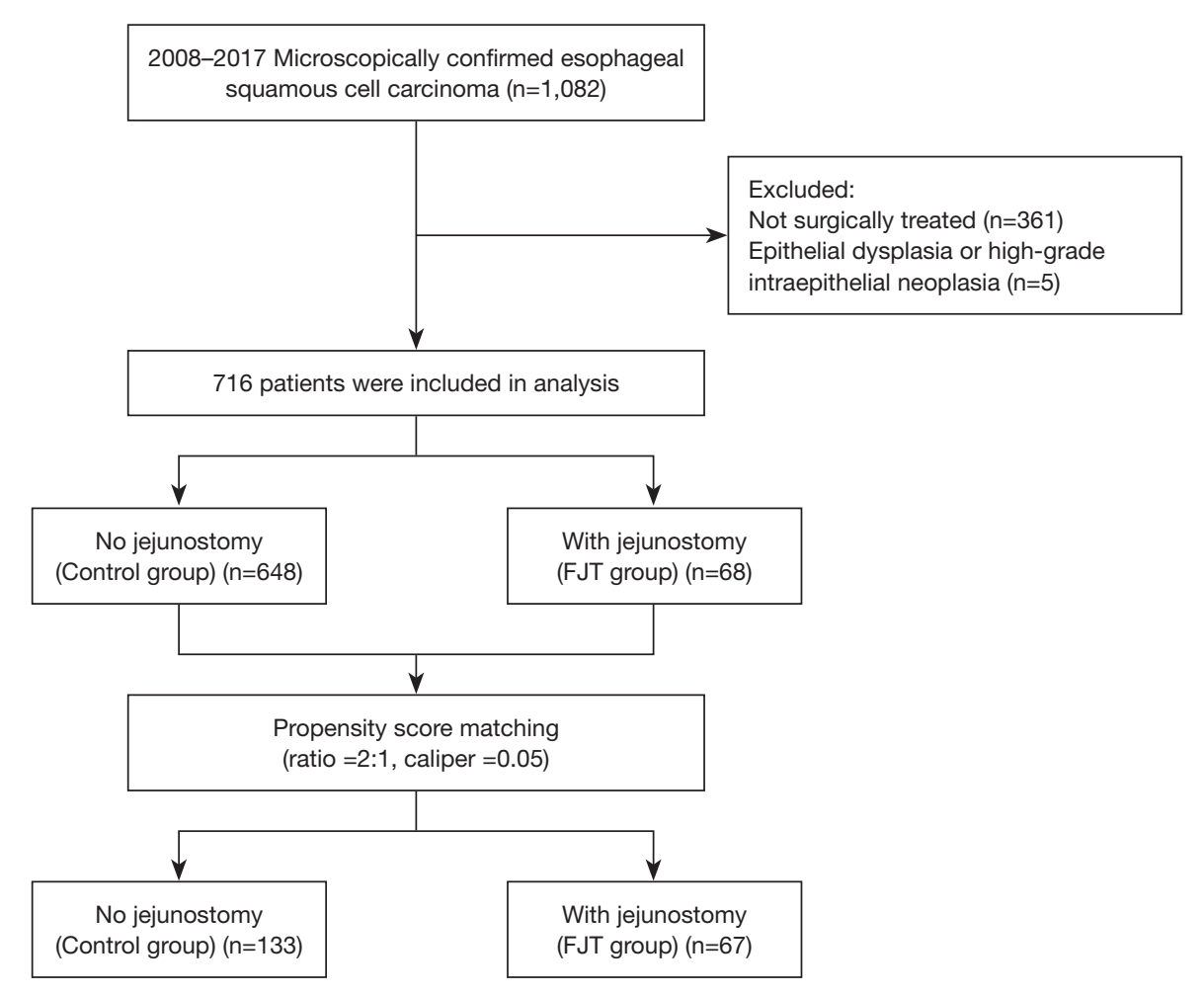

Figure 1 Diagram of patient selection and the procedure of propensity score matching.

offered to less than $20 \%$ of advanced stage patients in this study. Further assessments for eligibility of surgery were conducted after completion of at least 2 cycles of neoadjuvant therapy. Most patients in our institute received preoperative or intraoperative assessment to determine the necessity of a FJT. The following clinical characteristics were recognized as major risk factors for anastomotic leakage: (I) uncontrolled diabetes mellitus, with glycosylated hemoglobin (HbA1c) $\geq 8.0 \%$; (II) poor nutritional status, with body mass index (BMI) $<18.5 \mathrm{~kg} / \mathrm{m}^{2}$; (III) with neoadjuvant chemoradiotherapy; (IV) with intraoperative impairment of gastroepiploic artery. Minor risk factors included obesity, heavy smoking before surgery, upper thoracic ESCC and prolonged surgical duration, which was defined as longer than 6 hours after first incision. The patients with one major risk factors or more than one minor risk factors were suitable for intraoperative placement of FJT. All risk factors were retrospectively reviewed and collected from the EMR.

\section{Operation of esophagectomy and jejunostomy}

Surgical procedures included Sweet esophagectomy, Ivor Lewis esophagectomy, and McKeown esophagectomy, either with an open or minimally invasive approach. At minimum, a two-field lymphadenectomy was performed for all patients. A longitudinal Witzel jejunostomy technique was usually employed in selected patients with a higher presumed risk of anastomotic leakage as mentioned above. A feeding tube was inserted into the antimesenteric margin of jejunum approximately $20 \mathrm{~cm}$ distal to the Treitz ligament, with 4/0 Vicryl purse string suture around the site where the tube penetrated through the intestinal wall. A serosal tunnel was constructed for the catheter in the small intestine $3-5 \mathrm{~cm}$ proximally from the catheter's exit site using a continuous suture. The tube was then fixated to the peritoneum of internal abdominal wall and additionally to the skin with interrupted sealing sutures. All procedures were performed by the same surgical group in our department, which had ensured a stable quality of operation.

\section{Postoperative management and adjuvant therapies}

All patients with FJT (FJT group) initiated liquid enteral feeding on the morning of postoperative day 1 (POD1), using individualized formulas tailored by dieticians. Those 
without jejunostomy (control group) started with total parenteral nutrition (TPN) on POD1 with a stepwise increase of calories. Oral diet was usually resumed during POD5 to POD7 in the control group, unless there was any evidence of anastomotic leakage on contrast esophagram. In contrast, oral diet except for clear liquid was usually delayed for at least 3 days in the FJT group compared to the control group, to ensure the healing of anastomosis. Methods of catheter care were taught to the family caregivers before discharge of patients in the FJT group. The feeding tube was usually removed at the first follow-up visit (1 month after surgery) depending on the nutritional needs. Platinumbased adjuvant chemotherapy or chemoradiotherapy was offered to selected patients according to clinical guidelines.

\section{Patient follow-up}

First-year follow-up visits were scheduled at 1, 3, 6, 9, and 12 months after esophagectomy. Thereafter, patients would visit our outpatient clinic every 3 to 6 months for the following 4 years. Patients who did not comply with the follow-up plan would be contacted regularly by telephone to determine their vital status. The primary outcome of this study was overall survival (OS), which was calculated from the date of esophagectomy to the date of death. Other outcomes of interest included medical expenditure, length of postoperative hospital stay, time to recover from anastomotic leakage, short-term mortality, and postoperative complications. The time to recover from anastomotic leakage was calculated from the date of diagnosis of leakage to the date that the patient was discharged from hospital with closure of esophageal fistula itself and recovery of leakage-related complications. The last contact to the patients was January 31, 2020, and the median follow-up time of this study cohort was 67.3 months [range, 1 to 136 months; $95 \%$ confidence interval (CI), 62.37-72.23]. Patients who were lost to follow-up or survived through the cutoff date for follow-up were classified as censored data in the statistical analyses.

\section{Statistical analysis}

Patients were classified into the FJT group or control group according to the presence or absence of jejunostomy. Demographic and clinicopathologic data were summarized as frequency and percentage, with categorical variables and ordinal variables compared by Chi-square test and MannWhiney $\mathrm{U}$ test, respectively. Medical expenditure and length of postoperative hospital stay were presented as quartiles and compared by Mann-Whiney U test. Rate of postoperative mortality and complication was analyzed using a crosstable to calculate odd ratios (OR) and compared by Fisher's exact test. PSM was performed by the method of nearest neighbor in a 2:1 ratio, with a caliper of 0.05 (Figure 1). Potential confounding factors of survival outcome, such as age, sex, BMI, Charlson comorbidity index (CCI), pathological stage, neoadjuvant therapy, and adjuvant therapy were included in the calculation of propensity score. Survival curves were depicted by the Kaplan-Meier method and compared by log-rank test. Baseline variables that were considered clinically relevant or that showed a univariate relationship with OS were entered into multivariate Cox proportional hazards regression model. A two-sided $\mathrm{P}$ value $<0.05$ was considered statistically significant in all tests. All statistical analyses were performed in SPSS 23.0 software for Windows (IBM Corp., Armonk, NY, USA) except for PSM, which was conducted in R software version 3.6.3 (R Foundation for Statistical Computing, Vienna, Austria) with the "MatchIt" package.

\section{Results}

\section{Patient characteristics and risk of anastomotic leakage}

A total of 1,082 patients were assessed for eligibility, and 716 of them were finally included (Figure 1). Baseline characteristics and clinicopathologic information of all patients before and after PSM were summarized in Table 1 . In the overall study cohort, 68 out of 714 (9.5\%) patients received intraoperative placement of jejunostomy. The pathological stages were slightly different between the two groups $(\mathrm{P}=0.085)$, with a higher proportion of advanced stage (stage III and IV) patients in the FJT group (58.8\% vs. $44.0 \%)$. This was probably contributed by the significantly different distribution of pathological $\mathrm{N}$ stages $(\mathrm{P}=0.028)$. Otherwise, the FJT group and control group were similar regarding age, sex, BMI, CCI, tumor location, pathological $\mathrm{T}$ stage, tumor grade, surgical approach, as well as administration of neoadjuvant and adjuvant therapies, with all $\mathrm{P}$ values $>0.05$ (Table 1). After adjustment by PSM, 67 patients in the FJT group were matched to 133 patients in the control group, and all variables were balanced between groups, with all $\mathrm{P}$ values $>0.1$ (Table 1). Risk profile for anastomotic leakage in both groups is depicted and summarized in Table 2. The common risk factors observed in the FJT groups were uncontrolled diabetes mellitus 
Table 1 Characteristics of the patients who received esophagectomy for esophageal squamous cell carcinoma before and after propensity score matching

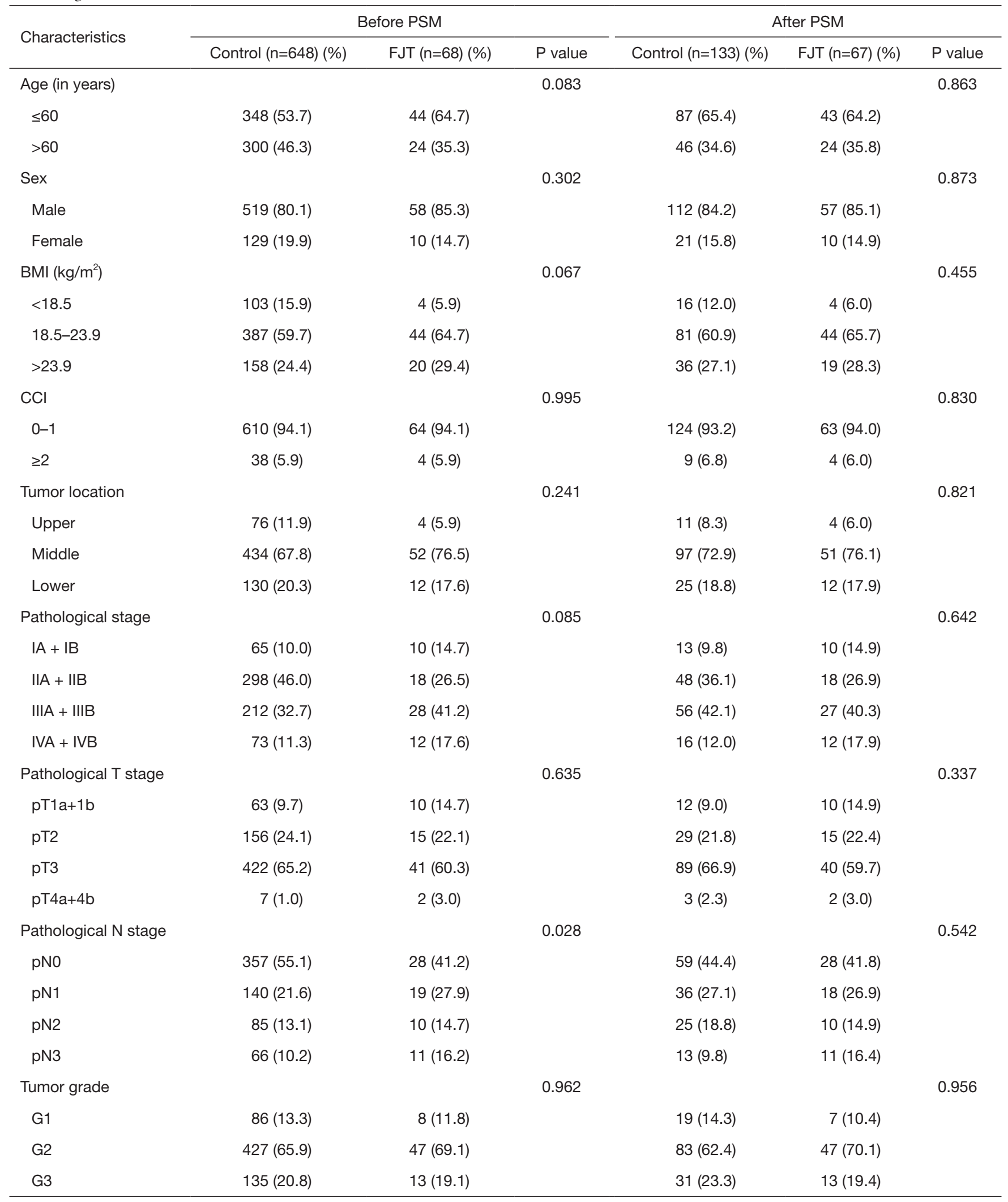

Table 1 (continued)

(C) Journal of Gastrointestinal Oncology. All rights reserved. 
Table 1 (continued)

\begin{tabular}{|c|c|c|c|c|c|c|}
\hline Characteristics & \multicolumn{3}{|c|}{ Before PSM } & \multicolumn{3}{|c|}{ After PSM } \\
\hline Neoadjuvant therapy & & & 0.359 & & & 0.989 \\
\hline Yes & $71(11.0)$ & $5(7.4)$ & & $10(7.5)$ & $5(7.5)$ & \\
\hline No & $577(89.0)$ & $63(92.6)$ & & $123(92.5)$ & $62(92.5)$ & \\
\hline Ivor Lewis & $95(14.7)$ & $8(11.8)$ & & $18(13.5)$ & $8(30.8)$ & \\
\hline McKeown & 475 (73.3) & $57(83.8)$ & & $98(73.7)$ & $56(83.6)$ & \\
\hline Surgical approach & & & 0.463 & & & 0.584 \\
\hline Yes & $259(40.0)$ & $32(47.1)$ & & $65(48.9)$ & $31(46.3)$ & \\
\hline No & $389(60.0)$ & $36(52.9)$ & & $68(51.1)$ & $36(53.7)$ & \\
\hline
\end{tabular}

${ }^{\text {a }}$, sweet esophagectomy: esophagectomy through a sole left thoracotomy incision. PSM, propensity score matching; FJT, feeding jejunal tube; $\mathrm{BMI}$, body mass index; $\mathrm{CCl}$, Charlson comorbidity index.

Table 2 Risk profile for anastomotic leakage in esophagectomy patients after propensity score matching

\begin{tabular}{|c|c|c|c|}
\hline Risk factors & Risk level & Control, $n=133(\%)$ & FJT, n=67 (\%) \\
\hline Uncontrolled diabetes mellitus & M & $2(1.5)$ & $28(41.8)$ \\
\hline Poor nutritional status & M & $6(4.5)$ & $3(4.5)$ \\
\hline Obesity & $\mathrm{m}$ & $0(0)$ & $2(3.0)$ \\
\hline Neoadjuvant chemoradiotherapy & M & $0(0)$ & $3(4.5)$ \\
\hline Upper thoracic ESCC & $\mathrm{m}$ & $11(8.3)$ & $4(6.0)$ \\
\hline \multicolumn{4}{|l|}{ Intraoperative risk factors } \\
\hline Intraoperative impairment of gastroepiploic artery & M & $0(0)$ & $2(3.0)$ \\
\hline \multicolumn{4}{|l|}{ Risk profile classification } \\
\hline No RF & & $108(81.2)$ & $14(20.9)$ \\
\hline $1 \mathrm{mRF}$ & & $15(11.3)$ & $6(9.0)$ \\
\hline$\geq 1 \mathrm{MRF}$, with or without $\mathrm{mRF}$ & & $8(6.0)$ & $36(53.7)$ \\
\hline$\geq 2$ mRFs, without MRF & & $2(1.5)$ & $11(16.4)$ \\
\hline
\end{tabular}

M, major; m, minor; RF, risk factor; mRF, minor risk factor; MRF, major risk factor; ESCC, esophageal squamous cell carcinoma; FJT, feeding jejunal tube. 
Table 3 Short-term results and complications after propensity score matching

\begin{tabular}{|c|c|c|c|c|}
\hline Short-term results & Control, n=133 (\%) & FJT, n=67 (\%) & Odds ratio ${ }^{a}$ & $P$ value \\
\hline Median (Q1, Q3) & $88,795(67,867,10,929)$ & $88,666(72,818,115,330)$ & - & 0.267 \\
\hline Length of postoperative hospital stay (d) & & & - & 0.802 \\
\hline Median (Q1, Q3) & $15(12,20)$ & $16(12,24.5)$ & & \\
\hline \multicolumn{5}{|l|}{ Short-term mortality } \\
\hline 90-day mortality & 0 & $1(1.5)$ & $3.015(2.475-3.673)$ & 0.335 \\
\hline 180-day mortality & $4(3.0)$ & $2(3.0)$ & $1.008(0.180-5.647)$ & 1.000 \\
\hline Pneumonia & $13(9.7)$ & 8 (11.9) & $1.025(0.923-1.137)$ & 0.222 \\
\hline Undesirable healing of surgical site & $9(6.8)$ & $5(7.5)$ & $1.071(0.516-2.227)$ & 1.000 \\
\hline Re-operation & $1(0.8)^{b}$ & $1(1.5)^{\mathrm{c}}$ & $1.500(0.370-6.082)$ & 1.000 \\
\hline Atrial fibrillation & $3(2.3)$ & $3(4.5)$ & $1.516(0.664-3.458)$ & 0.404 \\
\hline Complications in total $^{d}$ & $29(21.8)$ & $21(31.3)$ & $1.139(0.947-1.370)$ & 0.141 \\
\hline Jejunostomy-related & $0(0)$ & $2(9.5)$ & $1.105(0.962-1.270)$ & 0.171 \\
\hline Mean time to recover from anastomotic leakage (d) & 37.4 & 27.2 & - & 0.073 \\
\hline
\end{tabular}

(41.8\%), heavy smoking before surgery $(22.4 \%)$ and prolonged surgical time (19.4\%). Placement of a FJT was indicated in $70.1 \%$ patients in the FJT group, and $92.5 \%$ patients in the control group did not required intraoperative jejunostomy according to our conventional criteria.

\section{Fejunostomy and short-term impacts}

The short-term outcomes and complications after PSM are listed in Table 3. As can be seen, medical expenditure was approximately even between the FJT and control groups $(\mathrm{P}=0.267)$. There was no significant difference with regard to length of postoperative hospital stay $(\mathrm{P}=0.802)$. Proportions of delayed discharge from hospital ( $>30$ days) were $13.4 \%$ versus $12.0 \%$ [odds ratio $(\mathrm{OR})=1.016,95 \%$ $\mathrm{CI}, 0.907-1.138, \mathrm{P}=0.777$ ] in the FJT and control groups, respectively. Additionally, no statistically significant difference was found in terms of 90-day $(\mathrm{P}=0.335)$ or 180-day $(\mathrm{P}=1.000)$ mortality. From a statistical point of view, there was no significant increase of postoperative complications (OR $=1.139,95 \% \mathrm{CI}, 0.947-1.370, \mathrm{P}=0.141$ ) in the FJT group, which primarily comprised of anastomotic leakage (14.9\% vs. $11.3 \%$ ), pneumonia (11.9\% vs. $9.7 \%$ ), small bowel obstruction ( $1.5 \% v s .1 .5 \%)$, undesirable healing of incision site ( $7.5 \%$ vs. $6.8 \%)$, and re-operation ( $1.5 \%$ vs. $0.8 \%$ ). However, $90.5 \%$ of the complications were jejunostomy-unrelated in the FJT group. The mean time to recover from anastomotic leakage was 37.4 versus 27.2 days $(\mathrm{P}=0.073)$ in the control group and FJT group, respectively.

\section{fejunostomy and long-term survival outcomes}

The median OS of the FJT group and control group before PSM was 76.30 (95\% CI, 54.30-98.25) versus 62.10 (95\% CI, 51.50-72.70) months, with no statistically significant difference $(\mathrm{P}=0.390)$. After the adjustment of multiple 

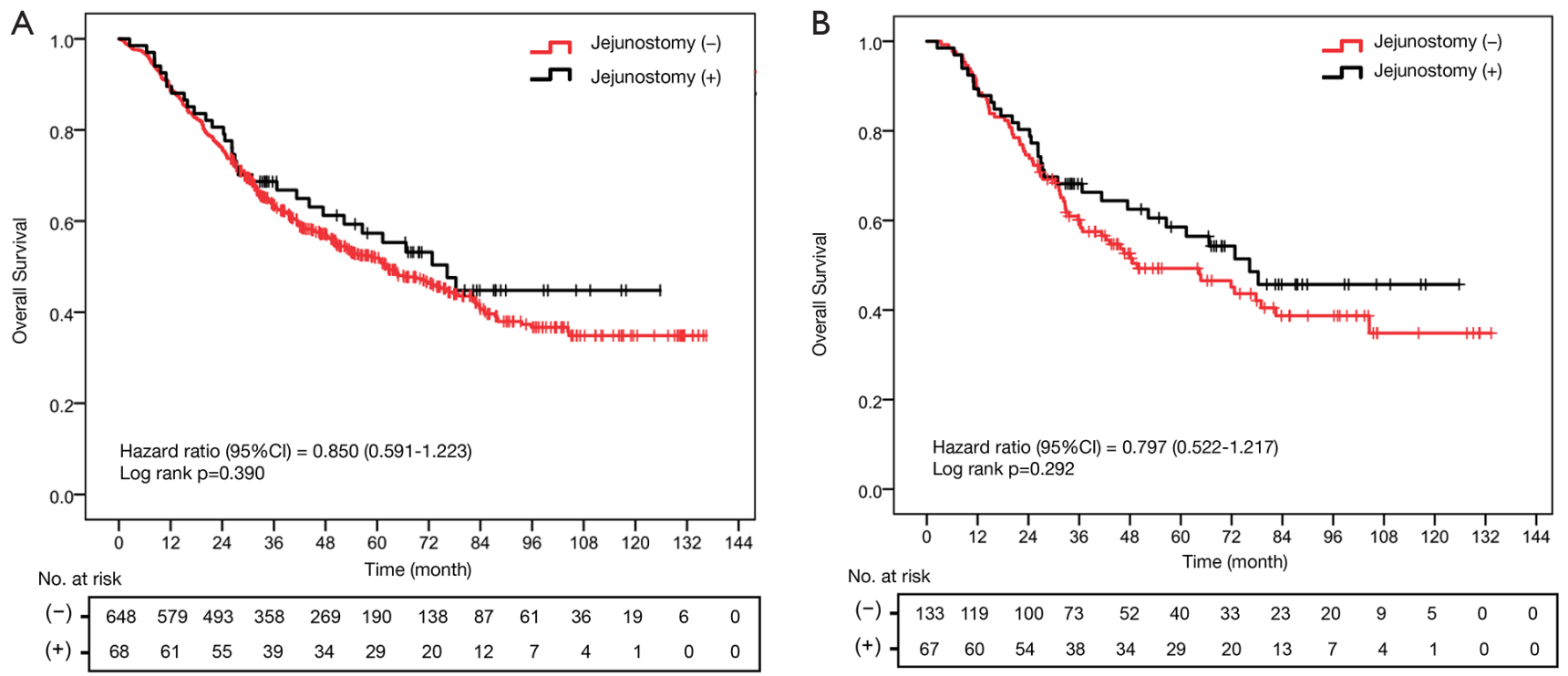

Figure 2 Overall survival curves in patients with or without jejunostomy after esophagectomy. (A) Survival curves before propensity score matching; (B) survival curves after propensity score matching.

survival confounding factors, there was still no significant difference between the FJT and control groups with respect to $\mathrm{OS}(\mathrm{P}=0.292)$ (Figure 2). In univariate analysis, nutritional jejunostomy was found to be an insignificant protective factor for OS [hazard ratio (HR) $=0.797,95 \%$ CI, $0.522-1.217, \log$ rank test $\mathrm{P}=0.292]$. Similarly, multivariate analysis adjusted for BMI, CCI, pathological stage, and adjuvant therapy did not reveal a worse OS in patients requiring nutritional jejunostomy $(\mathrm{HR}=0.716,95 \% \mathrm{CI}$, 0.464-1.106, $\mathrm{P}=0.132$ ) (Table 4).

\section{Subgroup analysis based on preoperative BMI}

A subgroup analysis stratified by preoperative BMI was conducted in PSM matched patients (Figure 3). Again, patients in FJT group that had a higher risk of anastomotic leakage achieved a comparable OS to the control group, regardless of the underweight (BMI $<18.5, \mathrm{P}=0.422$ ), normal weight $(\mathrm{BMI}=18.5-23.9, \mathrm{P}=0.480)$ or overweight (BMI $>23.9, \mathrm{P}=0.102$ ) patients. However, it should be noted that the results in the underweight and overweight patients might have been limited by their sample sizes.

\section{Discussion}

Nutritional status has been found to be associated with survival outcome in various cancers, especially cancers of the digestive system (17-19). Gastrointestinal malignancies are also demonstrated to have the highest prevalence of perioperative weight loss (3), and the implementation of nutritional support therapy (NST) appears to be a rational strategy in these circumstances. Postoperative EN is believed to be helpful in maintaining gastrointestinal integrity and avoiding complications associated with parenteral nutrition $(\mathrm{PN})$, such as central venous catheter infection and venous thrombosis (20). In this regard, EN is typically preferred over PN in real-world practice (21). FJT is most commonly used for NST in ESCC patients, and it is routinely performed in many institutions with the purpose of nutritional optimization (9). However, the distribution of FJT usage has only demonstrated a strong association with the traditions of operating centers rather than patient characteristics $(9,16)$. This may partially reflect the controversy and weakness of the scientific evidence for the role of FJT in the postoperative management of ESCC patients $(7-9,11,16)$.

For FJT users, increased incidence of complications, such as mechanical bowel obstruction, tube dislodgement, and site infection, has been reported in many studies $(11,22)$. These complications were associated with a higher frequency of emergency department visits (15). In several previous studies, the need for the routine placement of FJT has been put into doubt by weighting its nutritional advantage against its regimen-related morbidities and mortalities, along with 
Table 4 Univariate and multivariate analyses of overall survival in patients after propensity score matching

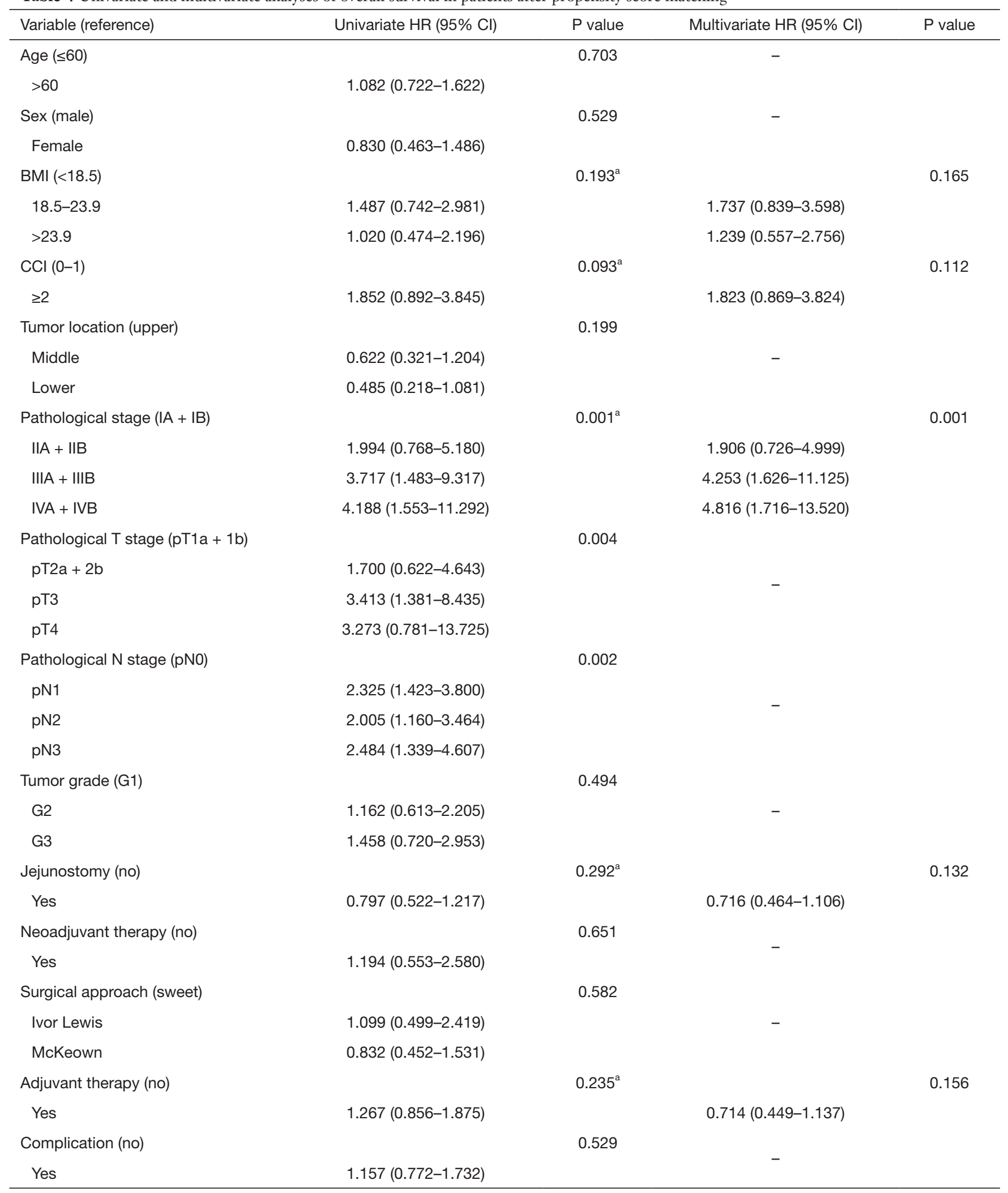

a , variables included in multivariate analysis. HR, hazard ratio; 95\% Cl, 95\% confidence interval; BMI, body mass index; CCI, Charlson comorbidity index.

(C) Journal of Gastrointestinal Oncology. All rights reserved. 

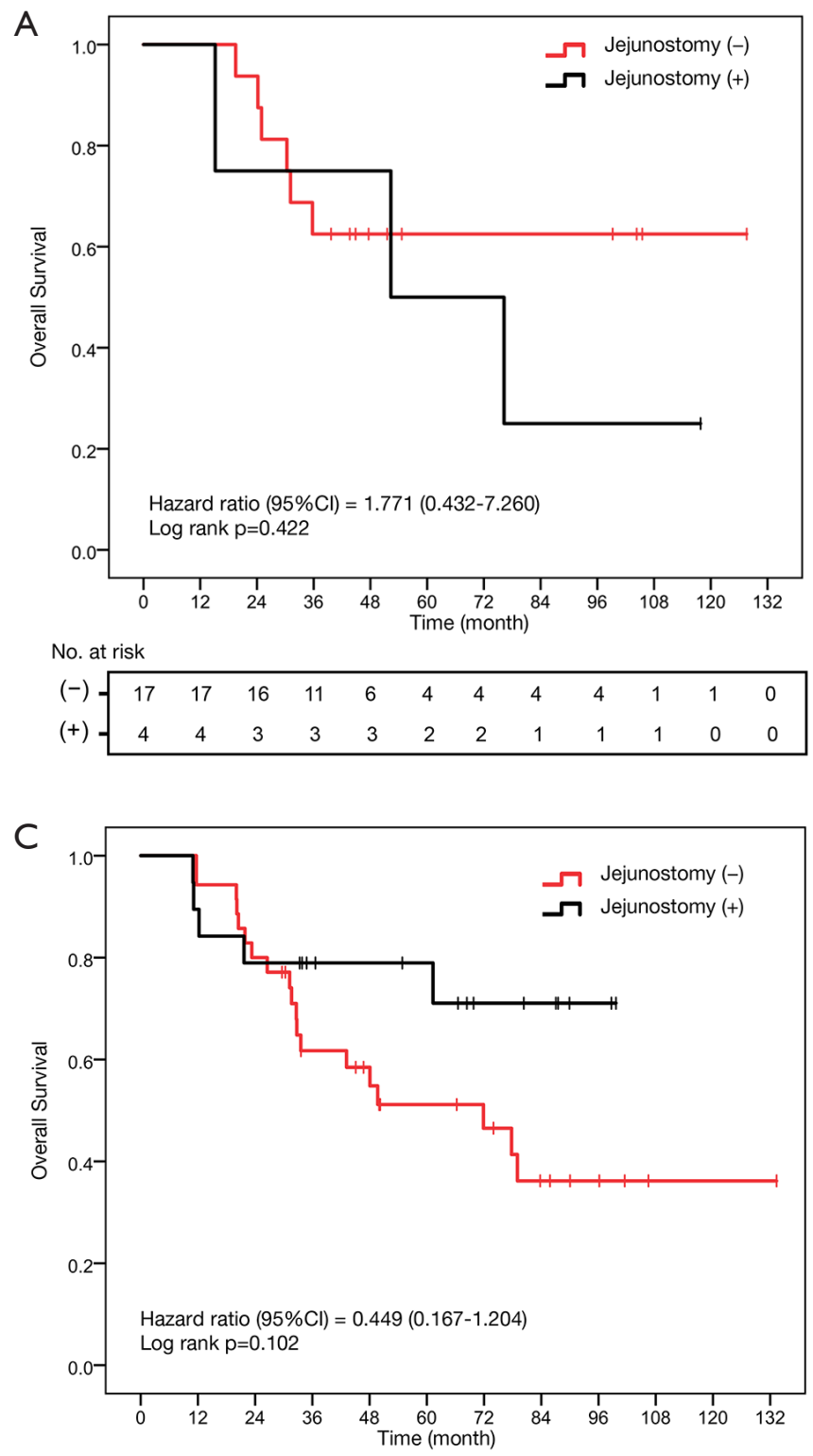

\begin{tabular}{|c|c|c|c|c|c|c|c|c|c|c|c|}
\hline \multicolumn{12}{|l|}{ No. at risk } \\
\hline$(-)-f$ & 34 & 29 & 20 & 17 & 12 & 10 & 6 & 4 & 1 & 1 & 0 \\
\hline$(+)$ & 17 & 15 & 12 & 11 & 10 & 6 & 5 & 2 & 0 & 0 & 0 \\
\hline
\end{tabular}

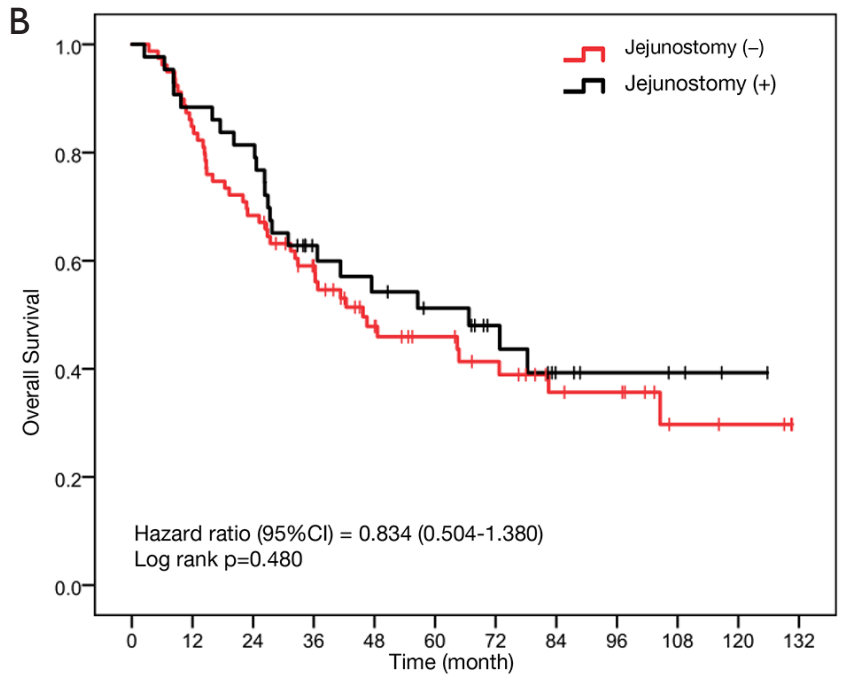

No. at risk

$\begin{aligned} & (+) \\
& (+)\end{aligned}-$\begin{tabular}{rrrrrrrrrrrr|r|}
80 & 68 & 55 & 42 & 28 & 22 & 18 & 12 & 10 & 4 & 3 & 0 \\
44 & 39 & 36 & 23 & 20 & 17 & 11 & 6 & 4 & 3 & 1 & 0 \\
\hline
\end{tabular}

Figure 3 Overall survival curves in propensity-score-matched patients with or without jejunostomy after being stratified by preoperative BMI. (A) Survival curves of underweight patients (BMI $\left.<18.5 \mathrm{~kg} / \mathrm{m}^{2}\right)$; (B) survival curves of normal weight patients $\left(\mathrm{BMI}=18.5-23.9 \mathrm{~kg} / \mathrm{m}^{2}\right)$; (C) survival curves of overweight patients $\left(\mathrm{BMI}>23.9 \mathrm{~kg} / \mathrm{m}^{2}\right)$.

its impact on HRQoL (7,23-25). Recently, a populationbased study of 8,632 patients confirmed the benefits of jejunostomy in reducing length of hospital stay $(\mathrm{OR}=0.78$, 95\% CI, 0.62-0.97, P=0.028), in-hospital mortality (OR $=0.76,95 \%$ CI, $0.63-0.90, \mathrm{P}=0.002)$ and 30-day mortality
(OR $=0.67,95 \%$ CI, $0.50-0.88, \mathrm{P}=0.004)$ (10). In the case of anastomotic leakage, FJT was found to be more suitable for longer administration of nutrients, which also accelerated the recovery of anastomotic leakage (26). This result was in consistent with the result of our study. Risk factors 
associated with anastomotic leakage usually include diabetes mellitus (27), higher preoperative HbA1c (28), active smoking (29), a poor nutritional status or obesity, etc. (27,30). In our study, patients receiving jejunostomy usually had a higher presumed risk of anastomotic leakage according to the assessment by surgeons, and therefore they could have been worse in terms of postoperative outcome (Table 2). However, they demonstrated a comparable medical expenditure, length of postoperative hospital stay, and similar safety profile to those without jejunostomy, which indicated the safety in performing this procedure (Table 3). Although safety is not a sufficient reason to recommend this procedure, any potential gain in nutritional benefit, HRQoL, or survival benefit should be assessed before considering this procedure. The patients in our study may benefit from jejunostomy to an extent, given their higher risk for anastomotic leakage but an approximate rate of incidence, which might be contributed by the preventive effect of FJT placement. As the nutritional indicators such as serum level of albumin, pre-albumin or transferrin usually fluctuate due to the perioperative intravenous infusions including the albumin itself, they were not an accurate reflection of the true nutritional status. Given this, we apply an indicator "mean time to recover from anastomotic leakage" as a surrogate to assess the postoperative nutritional benefit. It seems that placement of FJT could accelerate the recovery of anastomotic leakage in our study, with a statistically boundary significance (Table 3).

Despite the extensive research on the short-term outcomes of FJT, there is a scarcity of long-term assessments of FJT in patients after esophagectomy. For the first time, the current study assessed the long-term effects of jejunostomy by using PSM analysis. A more reliable conclusion could be expected after the adjustment of potential confounding factors. It has been reported that anastomotic leakage was associated with impaired OS and disease-free survival in both colonic and ESCAs $(31,32)$. In this study, no significant difference in survival outcome was found between the FJT group and control group either before or after PSM (Figure 2), which is consistent with the result of a previous study ( $\mathrm{HR}=0.89$, 95\% CI, 0.74-1.07, $\mathrm{P}=0.218$ ) (16). In other perspective, patients with a higher risk of anastomotic leakage achieved a comparable outcome to those of lower risk, which might indicate a potential positive effect of this procedure.

A clinical guideline formulated by the American Society for Parenteral and Enteral Nutrition recommends against the routine application of NST in patients undergoing major cancer operations (Grade A recommendation), based on the rationale that no improved outcome has been observed in many studies (21). However, its advantage may be uncovered when tailored to individual patient characteristics. For example, it is still recommended in highly selective patients with poorer general conditions, such as severe malnourishment (21). In our study, selected patients with a high presumed risk of anastomotic leakage can also benefit from the placement of FJT. Although a further subgroup analysis in our study did not show any significant survival benefit of FJT among patients with different levels of preoperative BMI, the evidence was not very conclusive in the underweight and overweight patients, given their limited sample sizes.

The results of this study were bolstered by its methodological strengths, which include prospectively gathered data and PSM analysis. This study is also limited by its retrospective nature, and thus the specific reasons for placement of FJT could not be ascertained in every single patients. Nevertheless, this potential selection bias was reduced by using PSM to adjust for multiple confounding factors. Additionally, inclusion of patients from only one institution, where chemotherapy alone was predominantly used instead of chemoradiotherapy, may limit the generalizability of the study results. However, all patients were operated on by the same surgical team, which likely reduced the heterogeneity in surgical techniques and postoperative management, and ensured comparability across patients.

\section{Conclusions}

In summary, this is the first study to use PSM analysis to evaluate the short-term and long-term outcomes of FJT in patients after esophagectomy. Patients with a high presumed risk of anastomotic leakage achieved comparable short-term and long-term outcomes in the presence of FJT. Intraoperative placement of FJT also appeared to accelerate the recovery of anastomotic leakage. Although the routine placement of FJT is not encouraged, its use in tailored patients should be considered, especially for those with a high risk of anastomotic leakage. Prospective study is warranted to further determine the preventive effect of FJT on esophagectomy-related complications.

\section{Acknowledgments}

The authors appreciate the academic support from AME Thoracic Surgery Collaborative Group. We thank Professor Shigeru Tsunoda for the critical comments and valuable advice on this study. 
Funding: None.

\section{Footnote}

Reporting Checklist: The authors have completed the STROBE reporting checklist. Available at http://dx.doi. org/10.21037/jgo-21-133

Data Sharing Statement: Available at http://dx.doi. org/10.21037/jgo-21-133

Conflicts of Interest: All authors have completed the ICMJE uniform disclosure form (available at http://dx.doi. org/10.21037/jgo-21-133). The authors have no conflicts of interest to declare.

Ethical Statement: The authors are accountable for all aspects of the work in ensuring that questions related to the accuracy or integrity of any part of the work are appropriately investigated and resolved. All procedures performed in this study involving human participants were in accordance with the Declaration of Helsinki (as revised in 2013). This study was approved by the Institutional Review Board of Guangdong Provincial People's Hospital (No. GDREC2019687H). Individual consent for this retrospective analysis was waived.

Open Access Statement: This is an Open Access article distributed in accordance with the Creative Commons Attribution-NonCommercial-NoDerivs 4.0 International License (CC BY-NC-ND 4.0), which permits the noncommercial replication and distribution of the article with the strict proviso that no changes or edits are made and the original work is properly cited (including links to both the formal publication through the relevant DOI and the license). See: https://creativecommons.org/licenses/by-nc-nd/4.0/.

\section{References}

1. Lagergren J, Smyth E, Cunningham D, et al. Oesophageal cancer. Lancet 2017;390:2383-96.

2. Kauppila JH, Johar A, Lagergren P. Postoperative Complications and Health-related Quality of Life 10 Years After Esophageal Cancer Surgery. Ann Surg 2020;271:311-6.

3. D'Journo XB, Ouattara M, Loundou A, et al. Prognostic impact of weight loss in 1-year survivors after transthoracic esophagectomy for cancer. Dis Esophagus 2012;25:527-34.
4. Berkelmans GHK, Fransen LFC, Dolmans-Zwartjes ACP, et al. Direct Oral Feeding Following Minimally Invasive Esophagectomy (NUTRIENT II trial): An International, Multicenter, Open-label Randomized Controlled Trial. Ann Surg 2020;271:41-7.

5. Sun HB, Li Y, Liu XB, et al. Early Oral Feeding Following McKeown Minimally Invasive Esophagectomy: An Openlabel, Randomized, Controlled, Noninferiority Trial. Ann Surg 2018;267:435-42.

6. Li X, Yan S, Ma Y, et al. Impact of Early Oral Feeding on Anastomotic Leakage Rate After Esophagectomy: A Systematic Review and Meta-analysis. World J Surg 2020;44:2709-18.

7. Álvarez-Sarrado E, Mingol Navarro F, J Rosellón R, et al. Feeding Jejunostomy after esophagectomy cannot be routinely recommended. Analysis of nutritional benefits and catheter-related complications. Am J Surg 2019;217:114-20.

8. Lorimer PD, Motz BM, Watson M, et al. Enteral Feeding Access Has an Impact on Outcomes for Patients with Esophageal Cancer Undergoing Esophagectomy: An Analysis of SEER-Medicare. Ann Surg Oncol 2019;26:1311-9.

9. Tham JC, Dovell G, Berrisford RG, et al. Routine use of feeding jejunostomy in oesophageal cancer resections: results of a survey in England. Dis Esophagus 2020;33:doz075.

10. Watson M, Trufan S, Benbow JH, et al. Jejunostomy at the time of esophagectomy is associated with improved short-term perioperative outcomes: analysis of the NSQIP database. J Gastrointest Oncol 2020;11:421-30.

11. Young MT, Troung H, Gebhart A, et al. Outcomes of laparoscopic feeding jejunostomy tube placement in 299 patients. Surg Endosc 2016;30:126-31.

12. Zheng R, Devin CL, Pucci MJ, et al. Optimal timing and route of nutritional support after esophagectomy: A review of the literature. World J Gastroenterol 2019;25:4427-36.

13. Holmén A, Hayami M, Szabo E, et al. Nutritional jejunostomy in esophagectomy for cancer, a national register-based cohort study of associations with postoperative outcomes and survival. Langenbecks Arch Surg 2020. [Epub ahead of print]. doi: 10.1007/s00423020-02037-0.

14. Akiyama Y, Iwaya T, Endo F, et al. Evaluation of the need for routine feeding jejunostomy for enteral nutrition after esophagectomy. J Thorac Dis 2018;10:6854-62.

15. Kidane B, Kaaki S, Hirpara DH, et al. Emergency department use is high after esophagectomy and feeding 
tube problems are the biggest culprit. J Thorac Cardiovasc Surg 2018;156:2340-8.

16. Klevebro F, Johar A, Lagergren J, et al. Outcomes of nutritional jejunostomy in the curative treatment of esophageal cancer. Dis Esophagus 2019;32:doy113.

17. $\mathrm{Wu} \mathrm{N}$, Chen G, Hu H, et al. Low pretherapeutic serum albumin as a risk factor for poor outcome in esophageal squamous cell carcinomas. Nutr Cancer 2015;67:481-5.

18. Tokunaga R, Sakamoto Y, Nakagawa S, et al. Prognostic Nutritional Index Predicts Severe Complications, Recurrence, and Poor Prognosis in Patients With Colorectal Cancer Undergoing Primary Tumor Resection. Dis Colon Rectum 2015;58:1048-57.

19. Li W, Li M, Wang T, et al. Controlling Nutritional Status (CONUT) score is a prognostic factor in patients with resected breast cancer. Sci Rep 2020;10:6633.

20. Oya H, Koike M, Iwata N, et al. Feeding duodenostomy decreases the incidence of mechanical obstruction after radical esophageal cancer surgery. World J Surg 2015;39:1105-10.

21. August DA, Huhmann MB; American Society for Parenteral and Enteral Nutrition (A.S.P.E.N.) Board of Directors. A.S.P.E.N. clinical guidelines: nutrition support therapy during adult anticancer treatment and in hematopoietic cell transplantation. JPEN J Parenter Enteral Nutr 2009;33:472-500.

22. Choi AH, O'Leary MP, Merchant SJ, et al. Complications of Feeding Jejunostomy Tubes in Patients with Gastroesophageal Cancer. J Gastrointest Surg 2017;21:259-65.

23. Scarpa M, Cavallin F, Noaro G, et al. Impact of jejunostomy during esophagectomy for cancer on health related quality of life. Chin J Cancer Res 2014;26:678-84.

24. Fenton JR, Bergeron EJ, Coello M, et al. Feeding jejunostomy tubes placed during esophagectomy: are

Cite this article as: Zhuang $\mathrm{W}, \mathrm{Wu} \mathrm{H}$, Liu $\mathrm{H}$, Huang $\mathrm{S}$, Wu Y, Deng C, Tian D, Zhou Z, Shi R, Chen G, Piessen G, Khaitan PG, Koyanagi K, Ozawa S, Qiao G. Utility of feeding jejunostomy in patients with esophageal cancer undergoing esophagectomy with a high risk of anastomotic leakage. J Gastrointest Oncol 2021;12(2):433-445. doi: 10.21037/jgo-21-133 they necessary? Ann Thorac Surg 2011;92:504-11; discussion 511-2.

25. Martin L, Lagergren J, Jia C, et al. The influence of needle catheter jejunostomy on weight development after oesophageal cancer surgery in a population-based study. Eur J Surg Oncol 2007;33:713-7.

26. Tao Z, Zhang Y, Zhu S, et al. A Prospective Randomized Trial Comparing Jejunostomy and Nasogastric Feeding in Minimally Invasive McKeown Esophagectomy. J Gastrointest Surg 2020;24:2187-96.

27. Hagens ERC, Reijntjes MA, Anderegg MCJ, et al. Risk factors and consequences of anastomotic leakage after esophagectomy for cancer. [Epub ahead of print]. doi: 10.1016/j.athoracsur.2020.08.022.

28. Okamura A, Yamamoto H, Watanabe M, et al. Association between Preoperative HbA1c Levels and Complications after Esophagectomy: Analysis of 15801 Esophagectomies from the National Clinical Database in Japan. Ann Surg 2020. [Epub ahead of print]. doi: 10.1097/ SLA.0000000000004547.

29. Van Daele E, Van de Putte D, Ceelen W, et al. Risk factors and consequences of anastomotic leakage after Ivor Lewis oesophagectomydagger. Interact Cardiovasc Thorac Surg 2016;22:32-7.

30. Kayani B, Okabayashi K, Ashrafian H, et al. Does obesity affect outcomes in patients undergoing esophagectomy for cancer? A meta-analysis. World J Surg 2012;36:1785-95.

31. Bashir Mohamed K, Hansen CH, Krarup PM, et al. The impact of anastomotic leakage on recurrence and long-term survival in patients with colonic cancer: A systematic review and meta-analysis. Eur J Surg Oncol 2020;46:439-47.

32. Aoyama T, Kazama K, Atsumi Y, et al. Clinical Influence of Anastomotic Leakage on Esophageal Cancer Survival and Recurrence. Anticancer Res 2020;40:443-9. 